\title{
Exotic hybrid mesons with light quarks
}

\author{
Claude Bernard, Tom Blum, ${ }^{\mathrm{b}}$ Thomas A. DeGrand, Carleton DeTar, ${ }^{\mathrm{d}}$ Steven Gottlieb, Urs M. Heller, \\ Jim Hetrick, ${ }^{\mathrm{g}}$ Craig McNeile, ${ }^{\mathrm{d}}$ Kari Rummukainen, ${ }^{\mathrm{e}}$ Bob Sugar, ${ }^{\mathrm{h}}$ Doug Toussaint ${ }^{\mathrm{g}}{ }^{*}$ and Matt Wingate ${ }^{\mathrm{c}}$ \\ ${ }^{a}$ Department of Physics, Washington University, St. Louis, MO 63130, USA \\ ${ }^{\text {b}}$ Department of Physics, Brookhaven National Lab, Upton, NY 11973, USA \\ ${ }^{c}$ Physics Department, University of Colorado, Boulder, CO 80309, USA

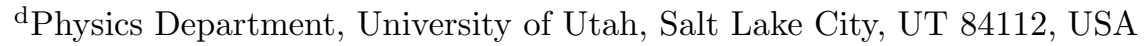 \\ ${ }^{\mathrm{e}}$ Department of Physics, Indiana University, Bloomington, IN 47405, USA \\ ${ }^{\mathrm{f}}$ SCRI, Florida State University, Tallahassee, FL 32306-4052, USA \\ ${ }^{\mathrm{g}}$ Department of Physics, University of Arizona, Tucson, AZ 85721, USA \\ ${ }^{\mathrm{h}}$ Department of Physics, University of California, Santa Barbara, CA 93106, USA
}

\begin{abstract}
Hybrid mesons, made from a quark, an antiquark and gluons, can have quantum numbers inaccessible to conventional quark-antiquark states. Confirmation of such states would give information on the role of "dynamical" color in low energy QCD. We present preliminary results for hybrid meson masses using light Wilson valence quarks.
\end{abstract}

\section{Introduction}

$\mathrm{QCD}$, the dynamical theory of strong interactions, appears to predict the existence of hadrons beyond those in the simple quark model, namely glueballs and hybrids. Confirmation of such states would give information on the role of "dynamical" color in low energy QCD. Hybrid mesons, made from a quark, an antiquark and one or more gluons, can have quantum numbers inaccessible to conventional quark-antiquark states. Isospin triplet hybrid mesons with such "exotic" quantum numbers are especially interesting because they cannot mix with conventional hadrons or with glueballs. Bound states of quarks and gluons are obviously nonperturbative phenomena, and in principle lattice gauge theory is the ideal method for calculating their properties, although in practice this turns out to be difficult.

The earliest lattice calculations of hybrid mesons used heavy quarks, where hybrid states

\footnotetext{
*presented by Doug Toussaint
}

appear as excitations of the gluonic string [1,2]. Here we present preliminary results for hybrid masses using lighter Wilson valence quarks, although not as light as the physical up and down quarks. The UKQCD collaboration has similar results for hybrid mesons 3 , 4 .

\section{Hybrid Operators}

To make an operator which creates a hybrid meson, we combine a quark, an antiquark and the color electric or magnetic field to form a color singlet with the desired spin, parity and charge conjugation. Because we do not include "quark-line disconnected" diagrams in our propagator, all our meson propagators are isospin triplets. The color electric and magnetic fields have $J^{P C}=1^{--}$and $1^{+-}$respectively. The spin, parity and charge conjugation from the quark and antiquark are those of the available quark bilinears listed here along with the corresponding mesons: (The $0^{+-}$ bilinear, $\bar{\psi} \gamma_{0} \psi$, is not expected to produce a par- 
ticle since it is the charge corresponding to a conserved current - the baryon number. We expect $\int d^{3} x \bar{\psi} \gamma_{0} \psi|0\rangle=0$.)

$$
\begin{array}{lll}
0^{++} & (\bar{\psi} \psi) & \left(a_{0}\right) \\
0^{+-} & \left(\bar{\psi} \gamma_{0} \psi\right) & (\mathrm{NONE}) \\
0^{-+} & \left(\bar{\psi} \gamma_{5} \psi, \bar{\psi} \gamma_{5} \gamma_{0} \psi\right) & (\pi) \\
1^{++} & \left(\bar{\psi} \gamma_{5} \gamma_{i} \psi\right) & \left(a_{1}\right) \\
1^{+-} & \left(\bar{\psi} \gamma_{5} \gamma_{0} \gamma_{i} \psi\right) & \left(b_{1}\right) \\
1^{--} & \left(\bar{\psi} \gamma_{i} \psi, \bar{\psi} \gamma_{i} \gamma_{0} \psi\right) & (\rho)
\end{array}
$$

We can also give the quark and antiquark a relative orbital angular momentum. This may be useful, because in the nonrelativistic quark model the $a_{1}\left(1^{++}\right)$, and hence the $0^{+-}$and $0^{--}$exotics constructed below, are $\mathrm{P}$ wave states. The operator $\stackrel{\leftrightarrow}{\partial}_{i}=\vec{\partial}_{i}-\overleftarrow{\partial}_{i}$ inserted in the quark bilinear brings in quantum numbers $1^{--}$, where the negative charge conjugation comes because $\mathrm{C}$ interchanges the quark and antiquark. Thus, a Pwave operator with $a_{1}$ quantum numbers, $1^{++}$, is $\epsilon_{i j k} \bar{\psi} \gamma_{j} \stackrel{\leftrightarrow}{\partial}_{k} \psi$. This operator may be advantageous because it couples the "large" components of the quark spinor to the large components of the antiquark spinor. For $F_{\mu \nu}$ we use a "pointlike" construction, illustrated here. Each open loop represents the product of the links, minus the adjoint of the product. Each of these links may actually be a "smeared" link, as illustrated on the right side of the figure.
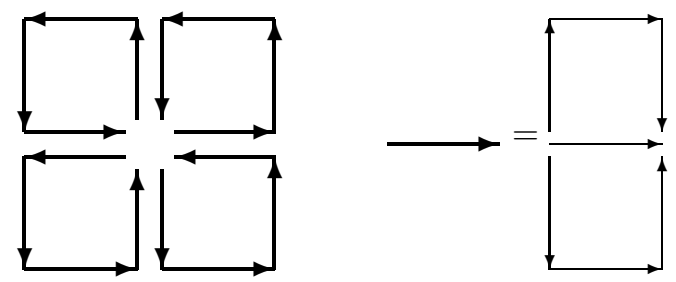

To make a color singlet including the octet $F_{\mu \nu}$ we take the color structure $\bar{\psi}^{a} F^{a b} \psi^{b}$, where $a$ and $b$ are color indices. We have computed propagators for the following hybrid operators:

$$
0^{-+}: \text {(Mixes with pion) }
$$

Take $1^{--}$quark bilinear and $\vec{B}$ :

$$
0^{-+}=\epsilon_{i j k} \bar{\psi}^{a} \gamma_{i} \psi^{b} F_{j k}^{a b}
$$

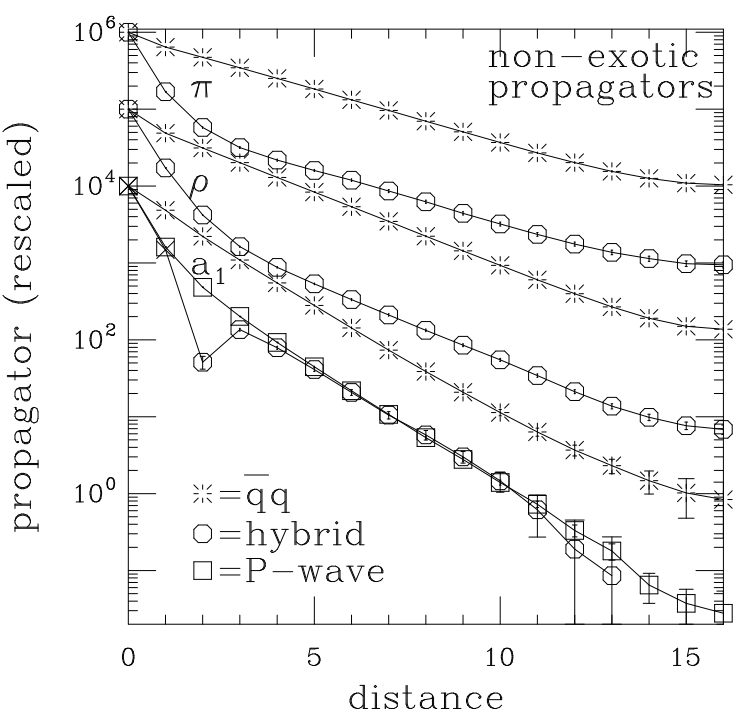

Figure 1. Propagators with conventional quantum numbers $0^{-+}=\pi, 1^{--}=\rho$ and $1^{++}=$ $a_{1}$. Propagators are scaled so that all propagators for the same quantum numbers begin at the same point. Hybrid propagators with these quantum numbers show the same mass (slope) as the quark-antiquark operators, indicating mixing between the hybrid and $\bar{q} q$ components. These propagators are for $\kappa=0.1585$.

$1^{--}$: (Mixes with rho)

Take $0^{-+}$quark bilinear and $\vec{B}$ :

$1^{--}=\epsilon_{i j k} \bar{\psi}^{a} \gamma_{5} \psi^{b} F_{j k}^{a b}$

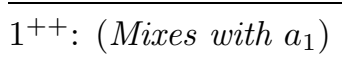

Take $1^{--}$quark bilinear and $\vec{E}$ :

$1^{++}=\epsilon_{i j k} \bar{\psi}^{a} \gamma_{j} \psi^{b} F_{0 k}^{a b}$

$$
0^{+-}:(\text {Exotic) }
$$

Take $1^{++}$quark bilinear and $\vec{B}$ :

$\mathrm{S}$ wave:

$0^{+-}=\bar{\psi}^{a} \gamma_{5} \gamma_{i} \psi^{b} B_{i}^{a b}=\bar{\psi}^{a} \gamma_{5} \gamma_{i} \psi^{b} \epsilon_{i j k} F_{j k}^{a b}$ 


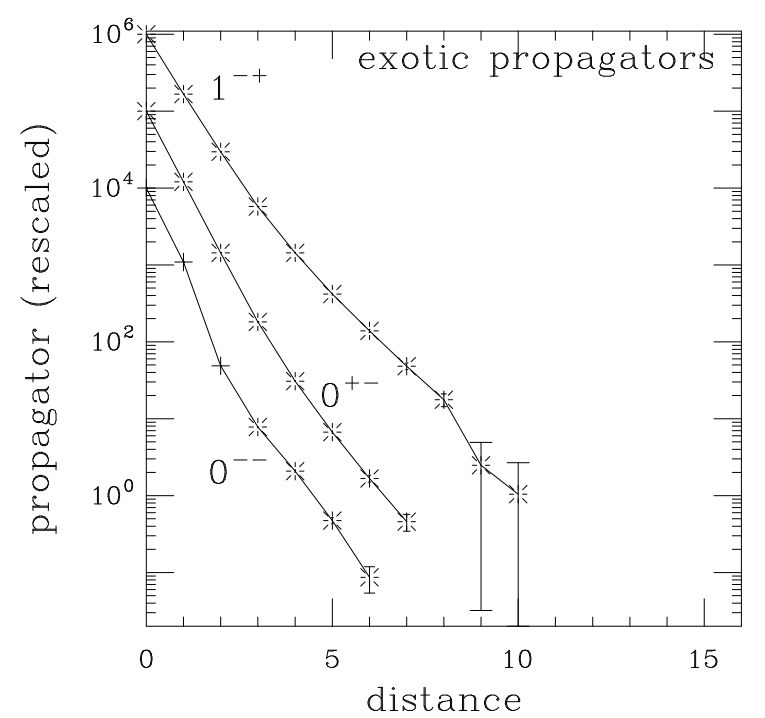

Figure 2. Hybrid propagators with exotic quantum numbers. These propagators use a twicesmeared $F_{\mu \nu}$ at $\kappa=0.1585$. Again, propagators have been rescaled to arbitrary values at distance zero. The plus signs in the $0^{--}$propagator indicate a change of sign. The scale is the same as Fig. 1 to facilitate comparison.

$\mathrm{P}$ wave:

$0^{+-}=\epsilon_{i j k} \bar{\psi}^{b} \gamma_{j} \stackrel{\leftrightarrow}{\partial}_{k} \psi^{a} B_{i}^{a b}=\bar{\psi}^{b} \gamma_{j} \stackrel{\leftrightarrow}{\partial}_{k} \psi^{a} F_{j k}^{a b}$

$0^{--}:($Exotic $)$

Take $1^{++}$quark bilinear and $\vec{E}$ :

$\mathrm{S}$ wave:

$0^{--}=\bar{\psi}^{a} \gamma_{5} \gamma_{i} \psi^{b} E_{i}^{a b}=\bar{\psi}^{a} \gamma_{5} \gamma_{i} \psi^{b} F_{i 0}^{a b}$

$\mathrm{P}$ wave:

$0^{--}=\epsilon_{i j k} \bar{\psi}^{b} \gamma_{j} \stackrel{\leftrightarrow}{\partial}_{k} \psi^{a} E_{i}^{a b}=\epsilon_{i j k} \bar{\psi}^{b} \gamma_{j} \stackrel{\leftrightarrow}{\partial}_{k} \psi^{a} F_{0 i}^{a b}(7)$

$1^{-+}:($Exotic $)$

take a $1^{--}$quark operator and $\vec{B}$ :

$1^{--} \otimes 1^{+-}=0^{-+} \oplus \mathbf{1}^{-+} \oplus 2^{-+}$

$1^{-+}=\epsilon_{i j k} \bar{\psi}^{a} \gamma_{j} \psi^{b} B_{k}^{a b}=2 \bar{\psi}^{a} \gamma_{j} \psi^{b} F_{j i}^{a b}$

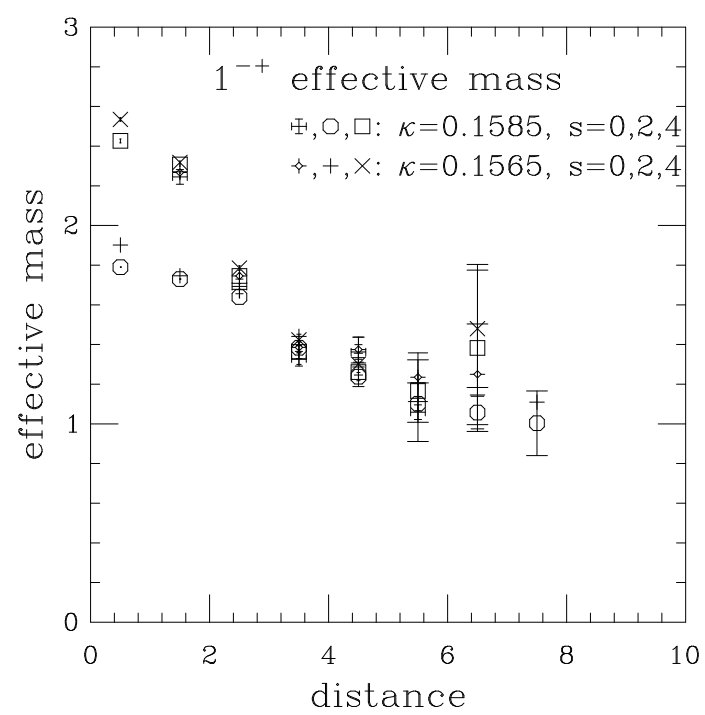

Figure 3. Effective mass for the $1^{-+}$propagator. "s" is the number of iterations of smearing in computing $F_{\mu \nu}$.

\section{Simulations}

Propagators were computed on a subset of the HEMCGC two flavor lattices. The lattice size is $16^{3} \times 32$, the sea quarks are Kogut-Susskind quarks with $a m_{q}=0.01$, and the gauge coupling is $6 / g^{2}=5.6$. We use Wilson valence quarks in computing propagators, with $\kappa=0.1565$ and 0.1585 . Many quantities have already been calculated on these lattices, including the conventional meson spectrum at these $\kappa[5]$. Estimates for the lattice spacing range from 1.8 to $2.4 \mathrm{GeV}$, depending on what quantity is chosen as the standard.

Conventional meson masses
$\kappa \quad a m_{\pi}$
$a m_{\rho}$
0.1565
$0.447(1)$
$0.526(3)$
$a m_{a_{1}}$
0.1585
$0.331(1)$
$0.442(4)$
$(12$

We used wall sources and point sinks, summed over space to get zero momentum, with four source slices per lattice. The exotic propagators shown here use $\mathrm{P}$-wave sources for $0^{+-}$and $0^{--}$, and an S-wave source for the $1^{-+}$.

We show hybrid propagators for conventional 


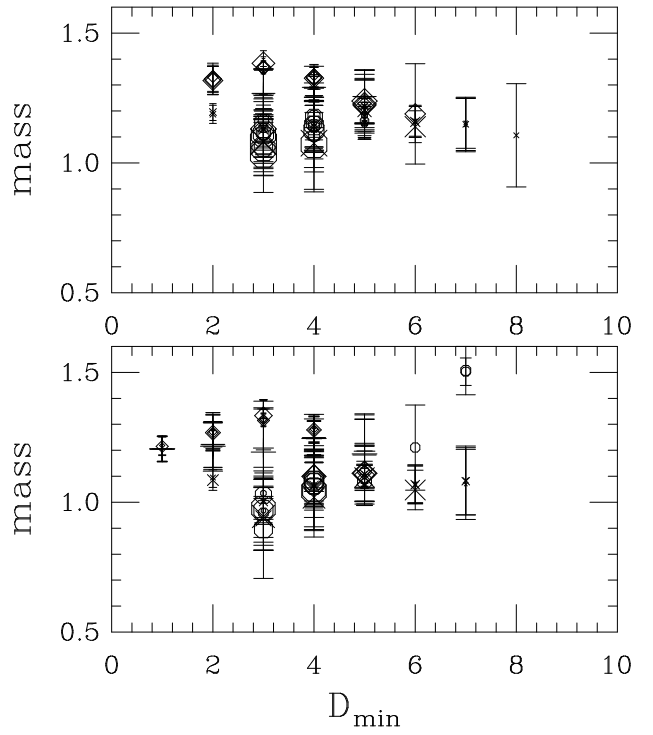

Figure 4. Mass fits for the $1^{-+}$propagator. We restrict to fits with confidence level greater than 0.1 and error less than 0.2. $\kappa=0.1565$ is on the top and $\kappa=0.1585$ on the bottom. Diamonds are for an unsmeared source, crosses for the smear $=2$ source, plusses for the smear $=4$ source, and octagons for the simultaneous two-propagator fits. Both one-mass and two-mass fits are included. The symbol size is proportional to the confidence level of the fit.

and exotic quantum numbers in Figs. 1 and 2. Of the three exotics studied, the $1^{-+}$is the lightest. Effective masses for the $1^{-+}$are in Fig. 3 . Although a better plateau in the effective mass is clearly desirable, we proceed to fit the propagator to an exponential over various ranges. The results are summarized in Fig. A. Of course, all our source operators (different smearings) should give the same masses, with different amplitudes depending on the overlap of the operator with the various states. To get mass estimates from shorter distances, we may try fitting two or more propagators simultaneously, constraining them to have the same masses but allowing different amplitudes[6]. Fig. [t also contains two source fits, with two masses and four amplitudes, using smearing levels 0 and 2 simultaneously, or 2 and 4 . The mass fits come out around $a m_{1^{-+}} \approx 2 a m_{1^{--}}$. If the valence quarks had the masses of the physical light quarks, this would be $\approx 2 a m_{\rho}$, but in fact the valence quarks are closer to the strange quark in mass.

\section{Conclusions}

The study of hybrid mesons on the lattice is in its infancy. These calculations combine the worst features of glueball and quark-antiquark spectrum calculations - operators with large fluctuations, with time consuming propagator inversions. The situation is like the early glueball calculations, with only short propagators available. We need more work to develop good operators, more statistics, and studies of dependence on quark mass and lattice spacing. Our results show that these calculations are doable, and we are optimistic about the ability of lattice calculations to find the spectrum of hybrid particles.

Calculations were done on the Intel Paragons at SDSC and Indiana University, the Alpha Cluster at PSC and the SP2 at CTC. This research was supported by the United States NSF and DOE.

\section{REFERENCES}

1. S. Perantonis and C. Michael, Nuc. Phys. B347, 854 (1990); L.A. Griffiths, C. Michael and P.E.L. Rakow, Phys. Lett. 129B, 351 (1983).

2. S. Catterall et al., Phys. Lett. B300, 393 (1993).

3. C. Michael, "Glueballs and Hybrid Mesons", hep-ph/9605243.

4. P. Lacock, C. Michael, P. Boyle and P. Rowland, "Orbitally excited and hybrid mesons from the lattice", hep-lat/9605025.

5. K. Bitar et al., Nucl. Phys. B (Proc. Suppl.) 30, 401 (1993); Phys. Rev. D 46, 2169 (1992); M. Wingate, T. DeGrand, S. Collins and U.M. Heller, Phys. Rev. D 52, 307 (1995).

6. See, for example, A. Duncan et al., Phys. Rev. D 51, 5101 (1994); D. Makovoz and G.A. Miller, Nuc. Phys. B468, 293 (1996). 